This is an open access article distributed under the CC BY-NC Attribution 4.0 International license.

\title{
Comparative Hepatic and Intestinal Efflux Transport of Statins ${ }^{\$}$
}

\author{
Feng Deng, ${ }^{1}$ Suvi-Kukka Tuomi, ${ }^{1}$ Mikko Neuvonen, Päivi Hirvensalo, Sami Kulju, \\ Christoph Wenzel, Stefan Oswald, Anne M. Filppula, and Mikko Niemi
}

Department of Clinical Pharmacology, Faculty of Medicine (F.D., S.-K.T., M.Ne, P.H., S.K., A.M.F., M.Ni.), and Individualized Drug Therapy Research Program, Faculty of Medicine, University of Helsinki, Helsinki, Finland (F.D., S.-K.T., M.Ne, P.H., S.K., A.M.F., M.Ni.); Institute of Pharmacology, Center of Drug Absorption and Transport, University Medicine Greifswald, Greifswald, Germany

(C.W., S.O.); Institute of Pharmacology and Toxicology, Rostock University Medical Center, Rostock, Germany (S.O.); and Department of Clinical Pharmacology, HUS Diagnostic Center, Helsinki University Hospital, Helsinki, Finland (M.Ni.)

Received February 19, 2021; accepted June 14, 2021

\section{ABSTRACT}

Previous studies have shown that lipid-lowering statins are transported by various ATP-binding cassette (ABC) transporters. However, because of varying methods, it is difficult to compare the transport profiles of statins. Therefore, we investigated the transport of 10 statins or statin metabolites by six ABC transporters using human embryonic kidney cell-derived membrane vesicles. The transporter protein expression levels in the vesicles were quantified with liquid chromatography-tandem mass spectrometry and used to scale the measured clearances to tissue levels. In our study, apically expressed breast cancer resistance protein (BCRP) and P-glycoprotein (P-gp) transported atorvastatin, fluvastatin, pitavastatin, and rosuvastatin. Multidrug resistance-associated protein 3 (MRP3) transported atorvastatin, fluvastatin, pitavastatin, and, to a smaller extent, pravastatin. MRP4 transported fluvastatin and rosuvastatin. The scaled clearances suggest that BCRP contributes to $87 \%-91 \%$ and $84 \%$ of the total active efflux of rosuvastatin in the small intestine and the liver, respectively. For atorvastatin, the corresponding values for P-gp-mediated efflux were $43 \%-79 \%$ and $66 \%$, respectively. MRP3, on the other hand, may contribute to $23 \%-26 \%$ and $25 \%-37 \%$ of total active efflux of atorvastatin, fluvastatin, and pitavastatin in jejunal enterocytes and liver hepatocytes, respectively. These data indicate that BCRP may play an important role in limiting the intestinal absorption and facilitating the biliary excretion of rosuvastatin and that P-gp may restrict the intestinal absorption and mediate the biliary excretion of atorvastatin. Moreover, the basolateral MRP3 may enhance the intestinal absorption and sinusoidal hepatic efflux of several statins. Taken together, the data show that statins differ considerably in their efflux transport profiles.

\section{SIGNIFICANCE STATEMENT}

This study characterized and compared the transport of atorvastatin, fluvastatin, pitavastatin, pravastatin, rosuvastatin, and simvastatin acid and four atorvastatin metabolites by six ABC transporters (BCRP, MRP2, MRP3, MRP4, MRP8, P-gp). Based on in vitro findings and protein abundance data, the study concludes that BCRP, MRP3, and P-gp have a major impact in the efflux of various statins. Together with in vitro metabolism, uptake transport, and clinical data, our findings are applicable for use in comparative systems pharmacology modeling of statins.

\section{Introduction}

Cardiovascular diseases are among the most common causes of death, accounting for approximately 17.9 million deaths worldwide in 2015 (Roth et al., 2017). 3-Hydroxy-3-methylglutaryl coenzyme A reductase inhibitors, also known as statins, are first-line drugs for primary and secondary prevention of cardiovascular diseases. Statins inhibit mevalonate production mediated by 3 -hydroxy-3-methylglutaryl

This work was supported by the ERC Consolidator Grant (Grant Agreement number 725249).

The authors declare no conflict of interest.

This work has not been presented in any meeting. Parts of this work are included in the Master's Thesis of Suvi-Kukka Tuomi.

${ }^{1}$ F.D. and S.-K.T. contributed equally.

https://dx.doi.org/10.1124/dmd.121.000430.

S This article has supplemental material available at dmd.aspetjournals.org. coenzyme A reductase, which is a key step in cholesterol biosynthesis. Inhibition of this enzyme leads to a reduced cholesterol production and increased expression of low-density lipoprotein cholesterol receptors in the liver (Slater and MacDonald, 1988). Ultimately, this results in a reduction in low-density lipoprotein cholesterol and triglyceride levels accompanied by decreased mortality and coronary events (Maron et al., 2000). Statins may also exert beneficial effects through a cholesterolindependent, pleiotropic manner by reducing systemic inflammation and platelet hyper-reactivity and improving endothelial function (Liao and Laufs, 2005). Although statins are widely used and generally accepted as efficient and safe (Yebyo et al., 2019), they may cause muscle toxicity ranging from mild and relatively common myalgia to rare but lifethreatening rhabdomyolysis (Harper and Jacobson, 2007).

Drug transporters play a key role in regulating drug levels in systemic circulation and various tissues (Giacomini et al., 2010). These proteins are located on the plasma membranes of cells, where they either pump their substrates into the cytosol or out of the cell. ATP-binding cassette

ABREVIATIONS: ABC, ATP-binding cassette; AUC, area under the plasma concentration-time curve; BCRP, breast cancer resistance protein; $\mathrm{CL}$, clearance; $\mathrm{CL}_{\mathrm{adj}}$, expression-adjusted clearance; $\mathrm{K}_{\mathrm{m}}$, Michaelis-Menten constant; $\mathrm{LC}-\mathrm{MS} / \mathrm{MS}$, liquid chromatography-tandem mass spectrometry; MRP, multidrug resistance-associated protein; OATP, organic anion-transporting polypeptide; P-gp, P-glycoprotein; SCHH; sandwichculture human hepatocyte; $V_{\max }$, maximal transport rate. 
(ABC) transporters are efflux pumps that use ATP to expel their substrates out of cells. Besides numerous drugs and other xenobiotics, they have an important task of excreting endogenous metabolites and waste products. P-glycoprotein (P-gp) and breast cancer resistance protein (BCRP) are among the most important efflux transporters, and they are followed by bile salt export pump and multidrug resistance-associated protein 2 (MRP2) (Hillgren et al., 2013). These transporters are abundantly expressed on the apical cell membranes in various pharmacokinetically relevant tissues, such as the intestine, liver, kidney, and bloodbrain barrier. There they limit the absorption and promote the elimination of a wide range of compounds. In contrast, the efflux transporters MRP3 and MRP4 are located on the basolateral cell membranes in the intestine and liver. These transporters pump their substrates toward systemic blood flow, thus promoting absorption and affecting the distribution of their substrates (van de Wetering et al., 2009; Kitamura et al., 2010a; Kitamura et al., 2010b).

In general, the statins have limited oral bioavailabilities due to marked first-pass loss (Neuvonen et al., 2006; Elsby et al., 2012). In the intestine, they are subject to biotransformation and apical efflux back to gut lumen. In the liver, they are extensively transported into hepatocytes by organic anion-transporting polypeptides (OATPs), which are important determinants of systemic exposure and clearance of the statins (Niemi et al., 2011). However, statin transport by the efflux transporters located on basolateral membranes in the intestine and liver are less well characterized. Since these transporters may also regulate the intracellular statin concentrations, their role in statin pharmacokinetics should be investigated in more detail. Hence, the present study aimed to compare the transport of atorvastatin, atorvastatin metabolites, fluvastatin, pitavastatin, pravastatin, rosuvastatin, and simvastatin acid by different efflux transporters using a vesicular transport assay.

\section{Materials and Methods}

Materials. Atorvastatin, atorvastatin-d5, 2-hydroxyatorvastatin, 2-hydroxyatorvastatin-d5, 2-hydroxyatorvastatin lactone, 2-hydroxyatorvastatin lactone-d5, 4-hydroxyatorvastatin, 4-hydroxyatorvastatin-d5, 4-hydroxyatorvastatin lactone, 4-hydroxyatorvastatin lactone- $\mathrm{d} 5$, fluvastatin- $\mathrm{d} 8$, pitavastatin- $\mathrm{d} 5$, pravastatin, pravastatin-d9, rosuvastatin, rosuvastatin- $\mathrm{d} 6$, and simvastatin acid-d6 were purchased from Toronto Research Chemicals (Toronto, Canada). Racemic fluvastatin, 3R,5S-fluvastatin, 3S,5R-fluvastatin, and pitavastatin were obtained from Santa Cruz Biotechnology (Dallas, TX). Simvastatin acid was purchased from SynFine Research (Ontario, Canada). Vesicles made of human embryonic kidney 293 cells were purchased from PharmTox at the Radboud University Medical Center (PharmTox, Radboud UMC, Nijmegen, The Netherlands). Solvents used in assays and analytical methods were of analytical quality and purchased from Sigma-Aldrich (St. Louis, MO). Ultrapure water for assays and analyses was purified using Milli-Q water purification system (Merck Millipore, Burlington, MA).

Vesicular Transport Assay. Vesicular transport assay was used to examine statin transport. The assays were performed essentially as described previously (Lehtisalo et al., 2020). In brief, transporter-expressing membrane vesicles (7.5 $\mu \mathrm{g}$ ) were preincubated at $37^{\circ} \mathrm{C}$ for 10 minutes in transport assay buffer (PharmTox, Radboud UMC, Nijmegen, The Netherlands) supplemented with $10 \mathrm{mM}$ $\mathrm{MgCl}_{2}$ and various concentrations of statins. After the preincubation, the transport was started by adding prewarmed Mg-ATP solution (final concentration 4 $\mathrm{mM}$ ) or distilled water to the wells. Solvent concentration in the assays were not more than $1.0 \%$ in screening and time-dependent transport studies and $1.5 \%$ in concentration-dependent transport studies. The reactions were incubated at $37^{\circ} \mathrm{C}$ for desired time, after which the transport was terminated with $200 \mu \mathrm{l}$ ice-cold stop buffer (PharmTox, Radboud UMC, Nijmegen, The Netherlands). Samples were then transferred onto a MultiScreenHTS FB Filter Plate $1.0 \mu \mathrm{m} / 0.65 \mu \mathrm{m}$ (Merck KGaA, Darmstadt, Germany), and the vesicles were washed thrice with $200 \mu \mathrm{l}$ stop buffer and twice with ice-cold washing buffer (40 mM 4-morpholinepropanesulfonic acid/Tris $\mathrm{pH} 7.0$ and $70 \mathrm{mM} \mathrm{KCl}$ ). After washing, the filter wells were air-dried, and the statins were eluted from vesicles with $50 \%$ methanol containing $25 \mathrm{ng} / \mathrm{ml}$ of isotope-labeled statin as an internal standard. All assays were performed in triplicates on 96-well plates.

Transport Studies. The vesicular transport assay and the function of membrane vesicles were verified by investigating the transport of estradiol-17-glucuronide and $N$-methyl-quinidine, known MRP and P-gp substrates, respectively. Statin transport was initially screened in BCRP, MRP2, MRP3, MRP4, MRP8, P-gp, and control vesicles: $10 \mu \mathrm{M}$ of atorvastatin, $3 R, 5 S$-fluvastatin, $3 S, 5 R$-fluvastatin, pitavastatin, pravastatin, and rosuvastatin and $1 \mu \mathrm{M}$ of simvastatin acid were incubated with the vesicles for 10 minutes. In addition, the transport of 10 $\mu \mathrm{M}$ atorvastatin metabolites was screened similarly in BCRP, MRP2, MRP3, MRP4, P-gp, and control vesicles. Statin-transporter combinations that exhibited notably higher uptake in the presence of ATP compared with that in the absence of ATP were further studied for time-linear range of transport with incubation times of 5, 10, and 15 minutes. Finally, the concentration-dependent transport (transporter kinetics) for confirmed substrates was determined in at least three separate experiments, in which the statin concentration ranged from 6 to $200 \mu \mathrm{M}$. The incubation time (5 minutes) was selected based on the results of the time-dependence experiments. Racemic fluvastatin was employed in the kinetic studies, since the fluvastatin enantiomers exhibited no selectivity in the investigated transporters and racemic mixture was more readily available.

$5^{\prime}$-Nucleotidase Activity Assay. The 5'-nucleotidase activity assay was used to determine the fraction of inverted membrane vesicles, essentially as described previously (Meszaros et al., 2011). In short, $15 \mu \mathrm{g}$ of transporter membrane vesicles were incubated at $37^{\circ} \mathrm{C}$ for 30 minutes with and without $3 \mathrm{mM}$ AMP and $0.3 \%$ Triton X-100 in $50 \mathrm{mM}$ Tris- $\mathrm{HCl}(\mathrm{pH} 7.4)$ and $4 \mathrm{mM} \mathrm{MgCl} 2$. The inorganic phosphate generated from AMP by $5^{\prime}$-nucleotidase located on the extracellular side of the plasma membrane was measured using Malachite Green Phosphate Detection Kit (R\&D Systems, Minneapolis, MN).

The $5^{\prime}$-nucleotidase activity was determined at four conditions: A) measurement with AMP and Triton X-100, which provides the maximum activity; B) measurement with AMP only, which provides the activity in right-side-out vesicles; C) measurement with Triton X-100 only, which shows the phosphate background in the presence of Triton X-100; and D) measurement without AMP and Triton X-100, which shows the background phosphate in the assay buffer.

$$
f_{\text {inverted }}=\frac{(A-C)-(B-D)}{A-C} * 100 \%
$$

The fraction of inverted membrane vesicles was calculated using eq. 1 . In the equation, A, B, C, and D indicate the amount of inorganic phosphate at the four conditions described above. A $-\mathrm{C}$ describes the total activity of $5^{\prime}$-nucleotidase, whereas $\mathrm{B}-\mathrm{D}$ describes the activity of $5^{\prime}$-nucleotidase in the right-side-out vesicles.

Analytical Methods. All statins and statin metabolites were analyzed using a Sciex 5500 Qtrap LC-MS/MS system (AB Sciex, Framingham, MA) interfaced with an ESI ion source. The chromatographic separation was performed on a Luna Omega polar C18 analytical column $(100 \times 2.1 \mathrm{~mm}$ i.d., $1.6 \mu \mathrm{m}$ particle size; Phenomenex, Torrance, $\mathrm{CA}$ ) protected by a guard column of the same material. The mobile phases $\mathrm{A}$ and $\mathrm{B}$ consisted of $5 \mathrm{mM}$ ammonium formate (pH 3.9, adjusted with 98\% formic acid) and acetonitrile, respectively, and the flow rate and the column temperature were maintained at $300 \mu 1 / \mathrm{min}$ and $40^{\circ} \mathrm{C}$. The following gradient conditions were applied: 1 minute at $20 \% \mathrm{~B}$ on hold, then a linear ramp from $20 \%$ B to $40 \%$ B over 3 minutes followed by a second linear ramp to $90 \%$ B over 2 minutes and 1 minute at $90 \% \mathrm{~B}$ before a re-equilibration step back to the initial conditions (20\% B). The mass spectrometer was operated in multiple reaction monitoring mode, and the characteristic ion transitions for each analyte and internal standard are presented in Supplemental Table S1.

Protein amounts (pmol/mg of protein) of BCRP, MRP2, MRP3, MRP4, and $\mathrm{P}$-gp in vesicles were quantified by mass spectrometry-based targeted proteomics using a validated LC-MS/MS method as described elsewhere (Gröer et al., 2013; Oswald et al., 2019). The isolated vesicles were subjected to determination of the whole protein concentrations using the BCA assay (Thermo Fisher Scientific, Schwerte, Germany). If necessary, membrane fractions were adjusted to a maximum protein amount of $2 \mathrm{mg} / \mathrm{ml}$. Subsequently, the membrane vesicles were directly digested with trypsin without further processing. In brief, $100 \mu \mathrm{l}$ of each membrane fraction was mixed with $10 \mu \mathrm{l}$ dithiothreitol $(200 \mathrm{mM}$, SigmaAldrich, Taufkirchen, Germany), $40 \mu 1$ ammonium bicarbonate buffer $(50 \mathrm{mM}$, $\mathrm{pH}$ 7.8, Sigma-Aldrich), and $10 \mu \mathrm{l}$ ProteaseMAX (1\%, m/v, Promega, 
Mannheim, Germany) and incubated for 20 minutes at $60^{\circ} \mathrm{C}$. After cooling down, $10 \mu$ iodoacetamide ( $400 \mathrm{mM}$, Sigma-Aldrich) were added, and the samples were incubated in a darkened water bath for 15 minutes at $37^{\circ} \mathrm{C}$. For protein digestion, $10 \mu \mathrm{l}$ trypsin (trypsin/protein ratio: 1/40, Promega) was added, and samples were incubated in a water bath for 16 hours at $37^{\circ} \mathrm{C}$. Digestion was stopped by addition of $20 \mu \mathrm{l}$ formic acid $(10 \% \mathrm{v} / \mathrm{v}$, Sigma-Aldrich). Afterward, the samples were centrifuged one more time for 15 minutes at $16,000 \mathrm{~g}$ and $4^{\circ} \mathrm{C}$. Then $50 \mu \mathrm{l}$ of the supernatant was mixed with $25 \mu \mathrm{l}$ isotope-labeled internal standard peptide mix $(10 \mathrm{nM}$ of each labeled peptide, Thermo Fisher Scientific). All sample preparation and digestion steps were performed using Protein LoBind tubes (Eppendorf, Hamburg, Germany). Protein quantification was conducted on a 5500 QTRAP triple quadrupole mass spectrometer (AB Sciex, Darmstadt, Germany) coupled to an Agilent Technologies 1260 Infinity system (Agilent Technologies, Waldbronn, Germany). Transporter proteins were simultaneously quantified using proteospecific peptides. Final protein abundance data (picomoles per milligram) were calculated by normalization to the total protein content of the isolated membrane fraction as determined by the BCA assay.

Data and Statistical Analysis. ATP-dependent transport was calculated by subtracting the statin uptake into vesicles in the absence of ATP from the statin uptake into vesicles in the presence of ATP. Uptake ratio was determined by uptake of investigated statin into vesicles in the presence of ATP divided by that in the absence of ATP. The ATP-dependent transport and uptake ratios in screening and time-dependent transport studies were compared with those in control vesicles using one-way ANOVA and Fisher's least significant difference analysis (GraphPad Software version 8.4, San Diego, CA). A $P$ value below 0.05 was considered statistically significant.

The kinetic parameters of statin transport were determined with GraphPad Prism 8.4. For these calculations, the mean ATP-dependent transport values of each concentration point from separate experiments were pooled, and these values were fitted to the Michaelis-Menten equation (eq. 2), wherein $v$ stands for the velocity of ATP-dependent transport, $\mathrm{V}_{\max }$ stands for the maximal transport rate, $[\mathrm{S}]$ stands for the substrate concentration, and $\mathrm{K}_{\mathrm{m}}$ stands for the MichaelisMenten constant.

$$
v=\frac{V_{\max }[S]}{K_{m}+[S]}
$$

The in vitro statin clearance was calculated from Michaelis-Menten parameters as shown in eq. 3 .

$$
C L_{\text {in vitro }}=\frac{V_{\max }}{K_{m}}
$$

The in vitro statin clearance was adjusted with vesicle protein expression as shown in eq. 4 ,

$$
C L_{a d j}=\frac{C L_{\text {in }} \text { vitro }}{\text { Protein expression } \text { in vitro } * f_{\text {inverted }}}
$$

in which $\mathrm{CL}_{\mathrm{adj}}$ stands for expression-adjusted clearance, Protein expression $n_{\text {in vitro }}$ stands for transporter abundance measured in vesicles, and $f_{\text {inverted }}$ stands for the fraction of membrane vesicles that is inverted. The clearance was further scaled to the tissue level by multiplying $\mathrm{CL}_{\text {adj }}$ with published transporter abundance data (eq. 5).

$$
C L_{\text {tissue }}=C L_{\text {adj }} * \text { Protein expression } \text { tissue }
$$

In eq. $5, \mathrm{CL}_{\text {tissue }}$ is the estimated tissue level efflux statin clearance, and Pro-

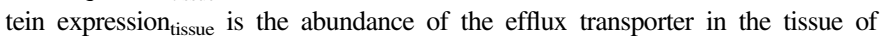
interest (Burt et al., 2016; Drozdzik et al., 2019).

\section{Results}

Transport of Known Substrates of MRP2, MRP3, MRP4, MRP8, and P-gp. The functionalities of the vesicles were verified using estradiol-17-glucuronide (50 $\mathrm{MM}$ for MRP2; $5 \mu \mathrm{M}$ for MRP3, MRP4, MRP8, and control vesicles) and $N$-methyl-quinidine ( $5 \mu \mathrm{M}$ for $\mathrm{P}$-gp) as positive controls. The uptake ratios of these probe substrates in MRP2, MRP3, MRP4, MRP8, and P-gp vesicles were 79, 38, 15, 2.8, and 2.4, respectively, verifying the functionality of the vesicles and vesicular transport assay (Supplemental Fig. S1).

Screening of Statin Efflux Transport. The screening of statin transport was conducted by incubating $10 \mu \mathrm{M}$ of statins (or $1 \mu \mathrm{M}$ in the case of simvastatin acid) with BCRP, MRP2, MRP3, MRP4, MRP8, Pgp, and control vesicles (Fig. 1; Supplemental Table S2). Atorvastatin was significantly transported by MRP3 (uptake ratio $2.2 \pm 0.6$ ) and P-gp (3.1 \pm 0.6$)$, and the uptake ratio of transport differed significantly from control (MRP3, $P=0.0102$; P-gp, $P<0.0001$ ). 3R,5S-Fluvastatin was significantly transported by BCRP $(3.8 \pm 0.9, P<0.0001)$ and P-gp $(2.8 \pm 0.3, P=0.0078)$, whereas $3 S, 5 R$-fluvastatin was significantly transported by BCRP $(3.1 \pm 1.6, P=0.0497), \mathrm{MRP} 3(3.2 \pm 1.0$, $P=0.0261)$, and MRP4 (3.1 $\pm 0.8, P=0.0458)$. Pitavastatin was clearly transported by BCRP $(4.6 \pm 0.6, P=0.0006)$ and P-gp $(3.3 \pm 1.0, P=$ $0.0306)$, and rosuvastatin was efficiently transported by BCRP $(8.4 \pm$ $3.1, P=0.0002$ ). For pravastatin and simvastatin acid, none of the uptake ratios differed significantly from the control.

In addition, we tested the transport of $10 \mu \mathrm{M}$ atorvastatin metabolites: 2-hydroxyatorvastatin, 4-hydroxyatorvastatin, 2-hydroxyatorvastatin lactone, and 4-hydroxyatorvastatin lactone in BCRP, MRP2, MRP3, MRP4, P-gp, and control vesicles. 2-Hydroxyatorvastatin was taken up in BCRP (uptake ratio $3.5 \pm 0.9, P=0.0279$ ) and MRP3 (3.3 \pm 0.6 , $P=0.0492$ ) vesicles significantly more than in control vesicles (uptake ratio $1.9 \pm 0.7$ ) (Fig. 1; Supplemental Table S3). The transport of 4-hydroxyatorvastatin in MRP3 $(4.4 \pm 1.5, P=0.0028)$ and P-gp (4.0 \pm $1.1, P=0.0076)$ vesicles differed significantly from control vesicles $(1.3 \pm 0.7)$. The 2 - and 4-hydroxyatorvastatin lactones showed no transport in any vesicles (Supplemental Table S3).

Time-Dependent Transport. The transport of statins by selected transporters was further investigated by studying the time-dependent transport (Supplemental Figs. S2-S7; Supplemental Tables S4-S5). In most cases, when notable transport was observed, the ATP-dependent transport plateaued already at 5 minutes as the statin uptake into vesicles and the escape of the statins due to passive diffusion reached equilibrium.

Atorvastatin transport was already prominent at 5 minutes in BCRP, MRP3, and P-gp vesicles with uptake ratios of $2.5(P=0.0012), 2.2$ $(P=0.0167)$, and $2.5(P=0.0019)$, respectively, which were significantly higher than that in control vesicles (Supplemental Fig. S2). On the other hand, the ATP-dependent transport and uptake ratio in MRP2 and MRP4 vesicles did not differ from the control vesicles, except for MRP2 at 10-minute time point.

The transport rates of both fluvastatin enantiomers in BCRP, MRP3, and P-gp vesicles were significantly higher than those in control vesicles at any given time, and the ATP-dependent transport was nearly plateaued 5 minutes after initiation of transport (Supplemental Figs. S3 and S4). The $3 R, 5 S$-fluvastatin transport rate in MRP2 and MRP4 vesicles was higher than that in control vesicles at 5 and 10 minutes. The $3 S, 5 R$-fluvastatin transport rate in MRP2 and MRP4 vesicles was higher than that in control vesicles only at 10 minutes. The transport rates of $3 R, 5 S$-fluvastatin and 3S,5R-fluvastatin in MRP8 vesicles differed significantly from those of control vesicles only at 5- and 10-minute time points, respectively.

Pitavastatin was transported by BCRP with high efficiency: The transport rates in BCRP vesicles were three times higher than those in MRP3 and P-gp vesicles (Supplemental Fig. S5). The transport rates in MRP3 vesicles were significantly higher than those of control at 5- and 15-minute time points, but only at 15 minutes the uptake ratio of 2.3 was significantly greater than in control vesicles $(P=0.0011)$. P-gp vesicles showed significantly higher transport rate $(P=0.0002)$ and the uptake ratio $(3.6, P=0.0004)$ at 5 -minute time point than control vesicles. 

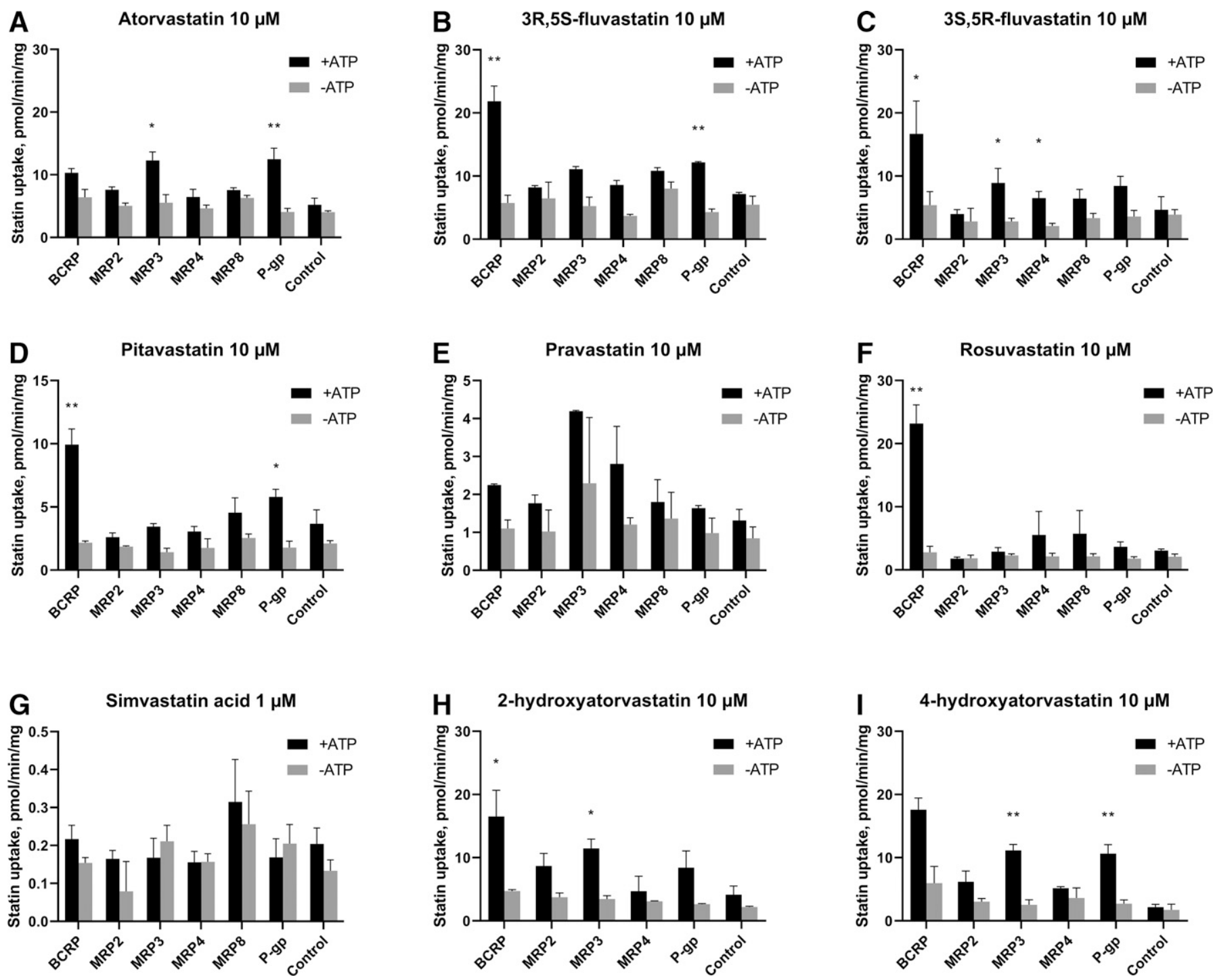

Fig. 1. The uptake of statins (A-G) and atorvastatin metabolites (H-I) in BCRP, MRP2, MRP3, MRP4, MRP8, P-gp, and control vesicles. Statin concentrations, incubation time, and amount of vesicles were $10 \mu \mathrm{M}$ (simvastatin acid $1 \mu \mathrm{M}$ ), 10 minutes, and $7.5 \mu \mathrm{g}$, respectively. Incubation time and the amount of vesicles in each experiment were 10 minutes and $7.5 \mu \mathrm{g}$, respectively. Black and gray bars represent statin uptake in vesicles in the presence and absence of ATP, respectively. Results are presented as mean \pm S.D. transport obtained from a single experiment performed with triplicate samples. One-way ANOVA was performed to evaluate whether the uptake ratio in transporter of interest differed from the control vesicles. ${ }^{*} P<0.05, * * P<0.01$.

In the screening, the uptake ratios of pravastatin were relatively high in BCRP, MRP3, and MRP4 vesicles, although they did not differ significantly from that of control vesicles. For this reason, the time-dependent transport of pravastatin was further studied. MRP3 exhibited low yet significant transport rates at 5 -minute $(P=0.0050)$ and 15 -minute $(P=0.0115)$ time points despite notable variation (Supplemental Fig. S6). In addition, the uptake ratio at 5 minutes $(2.2, P=0.0018)$ was significantly greater than that of control. Pravastatin transport rates in BCRP, MRP2, and MRP4 vesicles did not differ significantly from control, except for MRP2 at 15 minutes $(P=0.0473)$.

Rosuvastatin was transported by BCRP with high rate and uptake ratio at any given time, although the ATP-dependent transport plateaued already at 5 minutes (Supplemental Fig. S7). Although the MRP4 and P-gp transported rosuvastatin to some extent, the transport rates did not differ significantly from control vesicles and were approximately an order of magnitude lower than those in BCRP vesicles. Only the transport rate at 10 minutes in MRP4 vesicles was significantly greater than that in control vesicles $(P=0.0410)$. The high uptake ratio of rosuvastatin in MRP8 vesicles in screening studies was not replicated in the time-dependence studies.

The time-dependent transport of simvastatin acid was not further investigated, as the uptake ratios of simvastatin acid transport in the screening were relatively close to that in control vesicles.

Concentration-Dependent Transport (Transporter Kinetics). Based on atorvastatin screening and time-dependence studies, BCRP, MRP3, and P-gp were selected for kinetic measurements (Fig. 2). Atorvastatin had apparent affinities of 82.4, 31.5, and $10.7 \mu \mathrm{M}$, in BCRP, MRP3, and P-gp, respectively (Table 1). The maximum transport rate in the investigated transporters followed the same order, with the rate being the greatest for BCRP and the lowest for P-gp.

Racemic fluvastatin was employed in kinetic studies, since the enantiomers exhibited no distinct selectivity in the investigated transporters, and racemic mixture is more readily available. Racemic fluvastatin was well transported by BCRP and MRP3 with similarly high affinities $\left(\mathrm{K}_{\mathrm{m}}\right.$ 

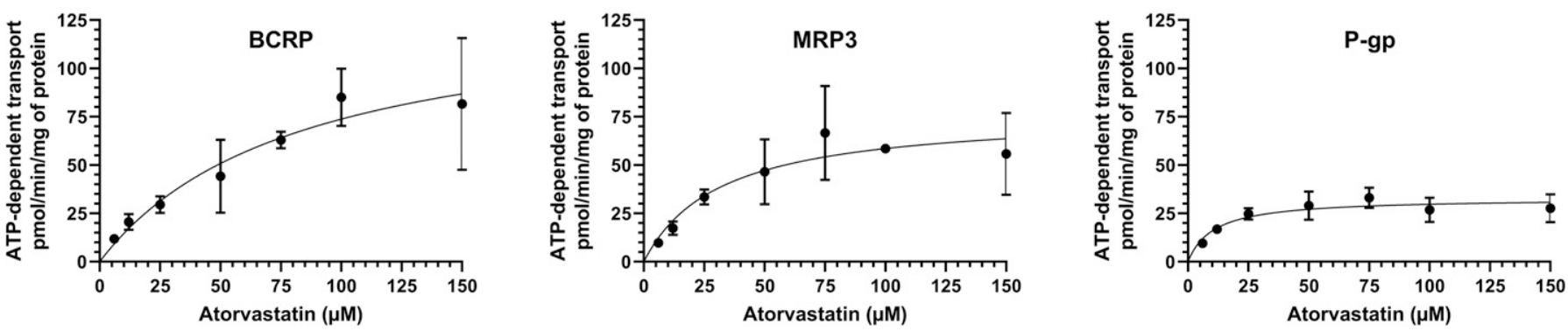

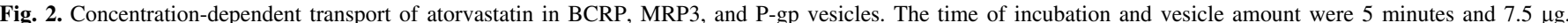

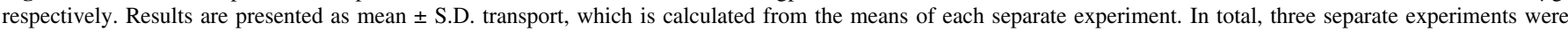
performed for each transporter with triplicate samples in each experiment.

25.8 and $48.9 \mu \mathrm{M}$, respectively), although the transport rate in BCRP was three times higher than that in MRP3 (Fig. 3; Table 1). In addition, MRP2, MRP4, MRP8, and P-gp vesicles were also capable of accumulating racemic fluvastatin in a concentration-dependent manner. Large deviation was observed in experiments despite the numerous replications, and this reflects the reliability of estimated Michaelis-Menten parameters. Therefore, only the curve fit and parameters of BCRP can be considered reliable. Nevertheless, the ATP-dependent transport was notably lower in control vesicles than in other vesicles. Furthermore, the uptake ratio in three control experiments was on average $1.26 \pm$ 0.15 , whereas the uptake ratio of MRP2 and P-gp, which had lowest ratio and highest deviation, respectively, were $1.81 \pm 0.44$ and $2.39 \pm$ 1.09 (Supplemental Table S6).

The apparent affinities of pitavastatin in BCRP, MRP3 and P-gp vesicles were high and relatively similar across the transporters $(16,9.0$, and $37 \mu \mathrm{M}$, respectively, Fig. 4). The maximum rate of transport in BCRP vesicles was four times higher than in other vesicles (Table 1). The MRP3-mediated transport of pitavastatin was less clear as the uptake ratio of $12 \mu \mathrm{M}$ pitavastatin in MRP3 vesicles remained below two $(1.8 \pm 0.2)$.

Pravastatin was transported in MRP3 vesicles only (Fig. 5). In the kinetic studies, pravastatin transport exhibited nonsaturable transport over the concentration range. Furthermore, the clearance of pravastatin was notably lower compared with the clearance of other statins in MRP3 (Table 1).

Rosuvastatin is a well established BCRP substrate with high transport rate and apparent affinity of transport. This was confirmed in our study with a single experiment, which concluded that $\mathrm{K}_{\mathrm{m}}$ of rosuvastatin transport in BCRP was approximately $4.2 \mu \mathrm{M}$ (Fig. 6). Meanwhile the apparent affinities of rosuvastatin in MRP4 and P-gp vesicles were 10fold lower ( $\mathrm{K}_{\mathrm{m}}$ of 39.3 and $46.2 \mu \mathrm{M}$, respectively). However, their transport rates in the presence of high concentrations of rosuvastatin showed large variability, which leads to the unreliable estimation of Michaelis-Menten parameters.

5 -Nucleotidase Activity and the Fraction of Inverted Membrane Vesicles. The fraction of inverted membrane vesicles was determined using the $5^{\prime}$-nucleotidase activity assay (Supplemental Fig. S8). The $\mathrm{f}_{\text {inverted }}$ of BCRP, MRP2, MRP4, MRP8, and P-gp vesicles were similar $(36 \%-52 \%)$, whereas the $f_{\text {inverted }}$ of MRP3 vesicles was higher $(65 \%)$.

Proteomic Measurements of Membrane Vesicles and the Estimation of Tissue-Specific Active Efflux Clearance. The transporter abundance in vesicle preparations was measured with a quantitative proteomic technique based on LC-MS/MS. The absolute abundances in BCRP, MRP2, MRP3, MRP4, and P-gp vesicles were 146, 85, 54, 44, and $59 \mathrm{pmol} / \mathrm{mg}$ of protein, respectively (Supplemental Table S7).

TABLE 1

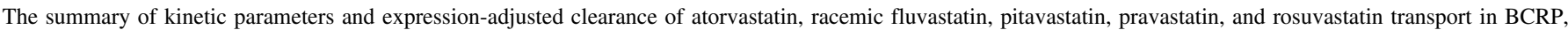
MRP2, MRP3, MRP4, MRP8, and P-gp vesicles.

The $95 \%$ confidence intervals $(95 \% \mathrm{CI})$ for the derived parameters are presented in parentheses.

\begin{tabular}{|c|c|c|c|c|c|}
\hline Statin & Transporter & $\mathrm{V}_{\max }(95 \% \mathrm{CI})$ & $\mathrm{K}_{\mathrm{m}}(95 \% \mathrm{CI})$ & $\mathrm{CL}_{\text {in vitro }}$ & $\mathrm{CL}_{\mathrm{adj}}$ \\
\hline & & $\mathrm{pmol} / \mathrm{min} / \mathrm{mg}$ & $\mu M$ & $\mu \mathrm{l} / \mathrm{min} / \mathrm{mg}$ & $\mathrm{nl} / \mathrm{min} / \mathrm{pmol}$ of transporter \\
\hline \multirow[t]{3}{*}{ Atorvastatin } & BCRP & $135(92.3-258)$ & $82.4(34.05-249)$ & 1.6 & 30.8 \\
\hline & MRP3 & $77.0(57.7-114)$ & $31.5(12.3-84.2)$ & 2.4 & 68.7 \\
\hline & P-gp & $32.9(27.3-40.0)$ & $10.7(4.34-23.1)$ & 3.1 & 133 \\
\hline \multirow[t]{6}{*}{ Fluvastatin } & BCRP & $408(313-580)$ & $25.8(9.81-68.4)$ & 15.8 & 298 \\
\hline & MRP2 & 105 (45.2-infinity) & 54.0 (1.92-infinity) & 1.9 & 49.9 \\
\hline & MRP3 & 170 (77.1-infinity) & 48.9 (2.12-infinity) & 3.5 & 97.6 \\
\hline & MRP4 & $54.7(23.4-4053)$ & 31.8 (0-infinity) & 1.7 & 76.4 \\
\hline & MRP8 & $74.5(41.6-580)$ & $73.0(10.5-1524)$ & 1.0 & $\mathrm{n} / \mathrm{a}$ \\
\hline & P-gp & 169 (51.6-infinity) & 282 (21.3-infinity) & 0.6 & 25.8 \\
\hline \multirow[t]{3}{*}{ Pitavastatin } & BCRP & $115(98.1-137)$ & $15.8(6.53-32.4)$ & 7.3 & 138 \\
\hline & MRP3 & $17.2(10.7-29.0)$ & $8.97(0-73.3)$ & 1.9 & 54.1 \\
\hline & P-gp & $31.8(18.3-97.4)$ & $36.6(3.64-316)$ & 0.9 & 37.4 \\
\hline Pravastatin & MRP3 & $\mathrm{n} / \mathrm{d}$ & $\mathrm{n} / \mathrm{d}$ & 0.2 & 5.6 \\
\hline \multirow[t]{3}{*}{ Rosuvastatin } & BCRP & $86.5(71.4-106)$ & $4.24(2.02-8.44)$ & 20.4 & 385 \\
\hline & MRP4 & 12.3 (5.13-infinity) & 39.3 (0.28-infinity) & 0.3 & 13.9 \\
\hline & P-gp & $19.1(11.3-51.8)$ & $46.2(5.7-344)$ & 0.4 & 17.8 \\
\hline
\end{tabular}



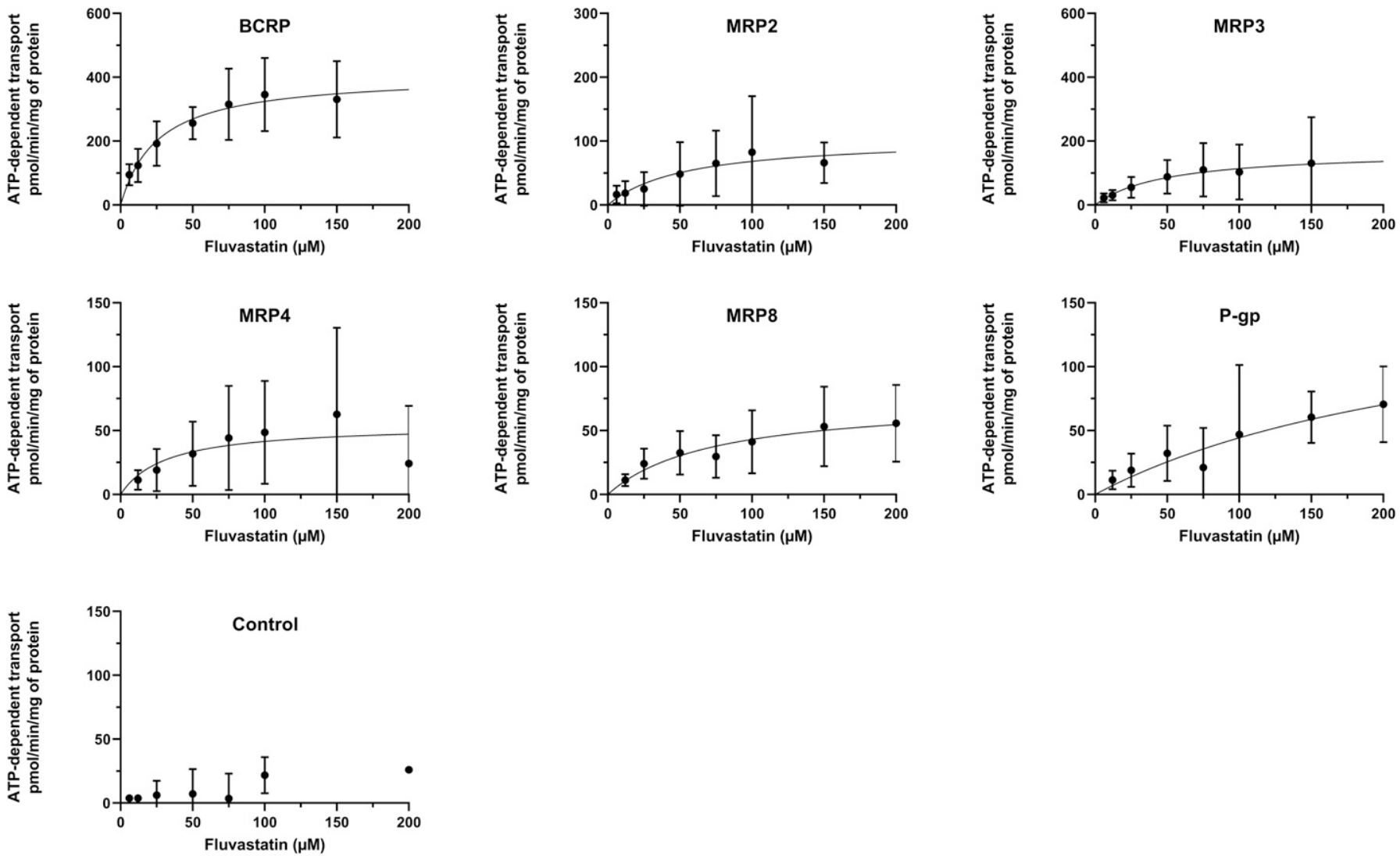

Fig. 3. Concentration-dependent transport of fluvastatin in BCRP, MRP2, MRP3, MRP4, MRP8, P-gp, and control vesicles. The time of incubation and vesicle amount were 5 minutes and $7.5 \mu \mathrm{g}$, respectively. Results are presented as mean \pm S.D. transport, which is calculated from the means of each separate experiment. In total, three separate experiments were performed for each transporter with triplicate samples in each experiment.

Based on the in vitro results, $5^{\prime}$-nucleotidase activity data, proteomics measurements, and published transporter abundance data from literature, we calculated the expression-adjusted clearance, $\mathrm{CL}_{\mathrm{adj}}$, (Table 1), and the individual contribution of efflux transporters to the total active efflux clearance in various tissues (Fig. 7; Supplemental Table S8). For atorvastatin, fluvastatin, and pitavastatin, basolateral pumps were estimated to
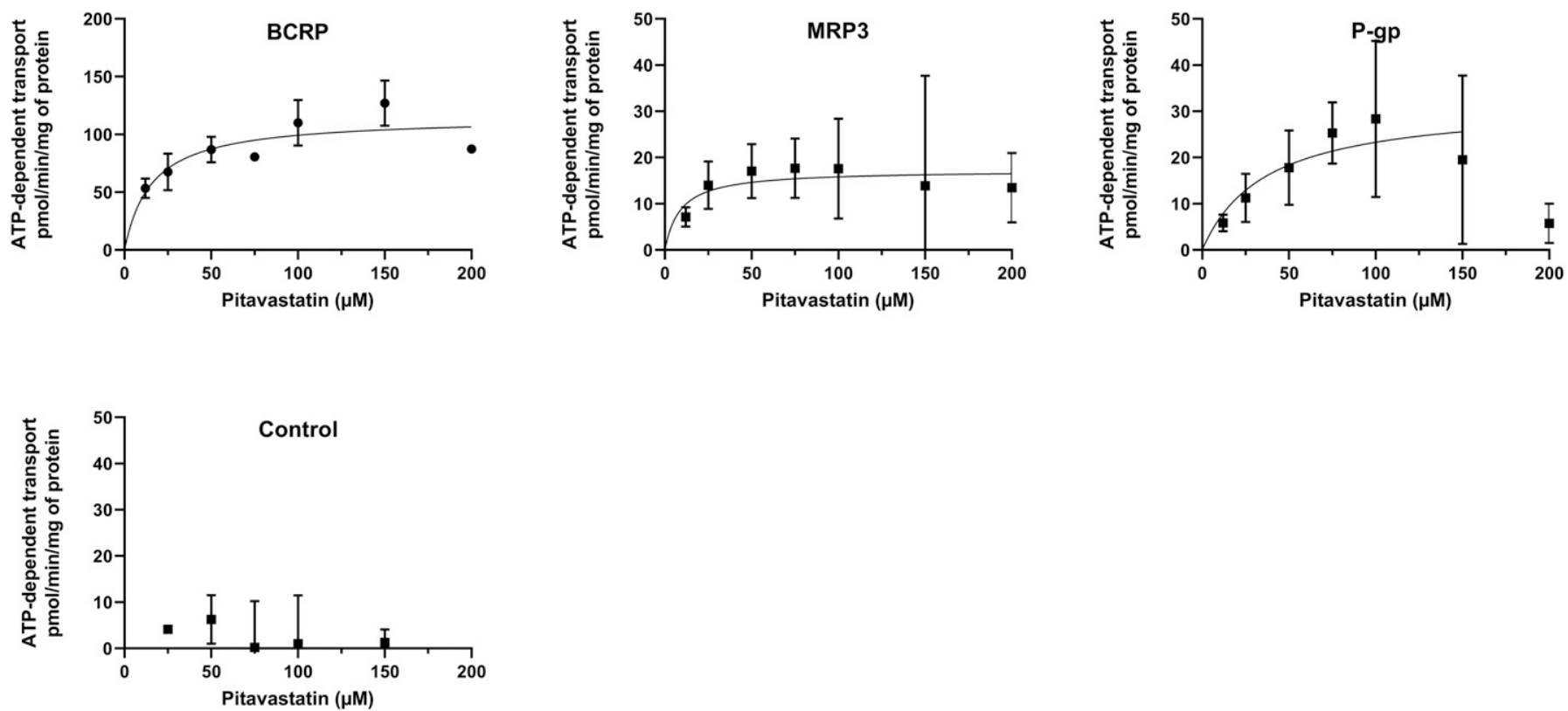

Fig. 4. Concentration-dependent transport of pitavastatin in BCRP, MRP3, P-gp, and control vesicles. The time of incubation and vesicle amount were 5 minutes and $7.5 \mu \mathrm{g}$, respectively. Results are presented as mean \pm S.D. transport, which is calculated from the means of each separate experiment. In total, three separate experiments (two for control vesicles) were performed for each transporter with triplicate samples in each experiment. 


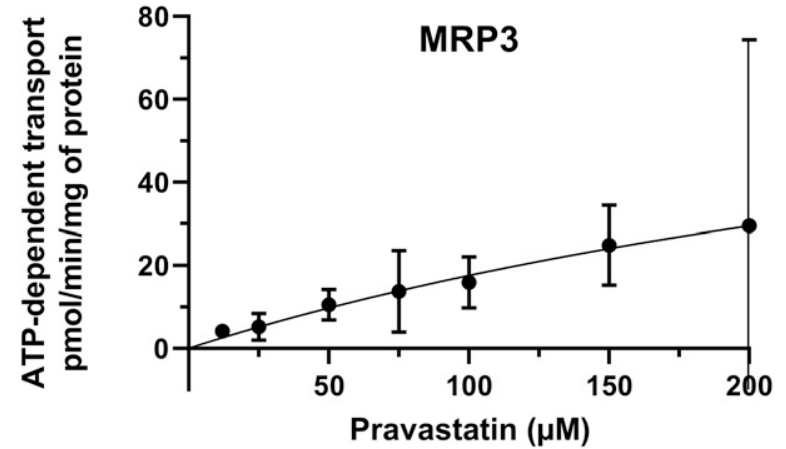

Fig. 5. Concentration-dependent transport of pravastatin in MRP3 vesicles. The time of incubation and vesicle amount were 5 minutes and $7.5 \mu \mathrm{g}$, respectively. Results are presented as mean \pm S.D. transport, which is calculated from the means of each separate experiment. In total, four separate experiments were performed with triplicate samples in each experiment.

contribute to approximately $50 \%$ and $28 \%$ of their efflux clearance in duodenal and jejunal enterocytes, respectively. Although the contribution of these transporters diminishes in the ileum, it still remains notable (approximately 15\%). Similarly in the hepatocytes, basolateral efflux, primarily mediated by MRP3, was estimated to contribute to one-third of the total known hepatic efflux of these statins. The scaled efflux clearance estimation underlines the impact of BCRP in rosuvastatin efflux, wherein over $84 \%$ of efflux clearance in both the intestine and liver was estimated to be mediated by BCRP. For atorvastatin, P-gp was estimated to comprise nearly $90 \%$ of its apical efflux clearance. Its role is particularly evident in the liver, in which P-gp is much more abundant than BCRP.

\section{Discussion}

In the present study, we evaluated the transport of six statins by six efflux transporters relevant for drug absorption, distribution, and elimination. This study is unique in that the transport was studied using uniform methods in a single laboratory, thus allowing comparison of statin efflux profiles. Of the apical efflux transporters tested, BCRP and P-gp transported atorvastatin, fluvastatin, pitavastatin, and rosuvastatin, and MRP2 transported fluvastatin. Interestingly, atorvastatin, fluvastatin, pitavastatin, and pravastatin were transported by the basolaterally expressed MRP3 and fluvastatin and rosuvastatin were expressed by MRP4. Simvastatin acid was not transported by any of the investigated transporters. This could be due to high passive permeability, which may result in a false negative result in the vesicular transport assay.
Furthermore, kinetic parameters from membrane vesicle assays combined with protein expression data, allowed the scaling of the obtained findings to tissue level. Taken together, our data indicate that statins differ in their efflux transport profiles.

BCRP, MRP2, and P-gp are expressed on the apical plasma membranes in tissues important for pharmacokinetics. There, they limit the absorption and facilitate the excretion of their substrates. Previous pharmacogenetic studies have suggested that BCRP restricts the intestinal absorption of atorvastatin, fluvastatin, and rosuvastatin (Keskitalo et al., $2009 \mathrm{~b}, \mathrm{c})$. Indeed, we observed extensive BCRP-mediated transport of fluvastatin, pitavastatin, and rosuvastatin, and minor but significant transport of atorvastatin. The more lipophilic pitavastatin and especially atorvastatin were good P-gp substrates, whereas the hydrophilic rosuvastatin was poorly transported by P-gp. Interestingly, all the statins transported by P-gp contain at least one nitrogen atom, which could be beneficial when interacting with the negatively charged binding cavity of P-gp (Deng et al., 2020).

MRP3 and MRP4 are expressed on the basolateral plasma membrane in small intestine and liver (Drozdzik et al., 2019). According to a recent meta-analysis, the hepatic abundance of MRP3 in healthy Caucasian adults is similar to that of P-gp and $40 \%$ lower than that of MRP2 (Burt et al., 2016). Furthermore, when all the ethnicities and disease states were included in the meta-analysis, the hepatic abundance of MRP4 was 70\% lower than that of MRP3 and similar to the level of BCRP. In the enterocytes, they may facilitate the vectorial movement of their substrate drugs and metabolites by flipping them from the enterocyte to mesenteric blood (van de Wetering et al., 2009; Kitamura et al., 2010a; Kitamura et al., 2010b; Proctor et al., 2016). Our estimations of active efflux clearance suggest that this basolateral pathway may be important for the absorption of several statins (Fig. 7). In the liver, statins are actively taken into the hepatocytes by OATP1B1, OATP1B3, and OATP2B1 (Bi et al., 2019). MRP3 and MRP4 may partly counteract this by returning statins from hepatocytes back to the sinusoidal blood. We hypothesize that basolateral transporters may additionally promote statin elimination and pharmacological activity by distributing statins and their metabolites more evenly in the hepatocytes along the sinusoids. This hepatocyte hopping could prevent the saturation of the pharmacological target, metabolic enzymes, and biliary efflux transporters (Iusuf et al., 2012). Based on our scaled clearance values, MRP3 could affect the intracellular statin efflux and enable hepatocyte shuffle for statins.

Atorvastatin is a lipophilic statin with an oral bioavailability of $14 \%$, which is explained by first-pass hepatic extraction (Lennernäs, 2003). Here, the transport kinetics of atorvastatin and its metabolites were determined for the first time using membrane vesicles. The highest affinity and abundance-scaled clearance of atorvastatin were observed
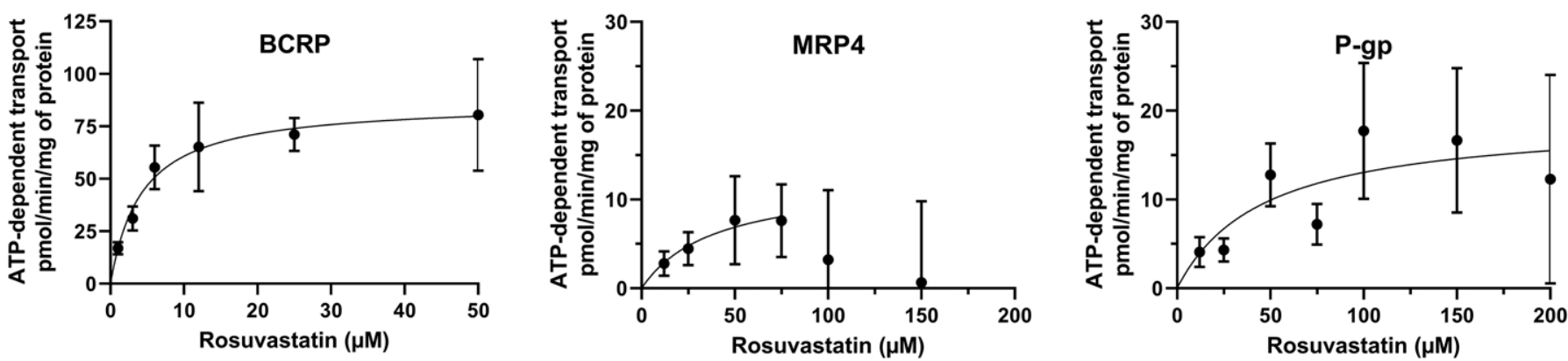

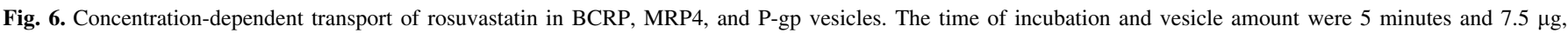

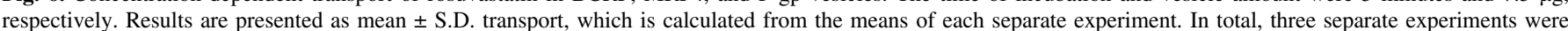

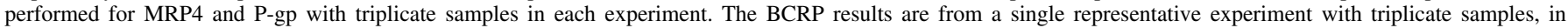
which data are presented as mean \pm S.D. transport. 
Duodenum

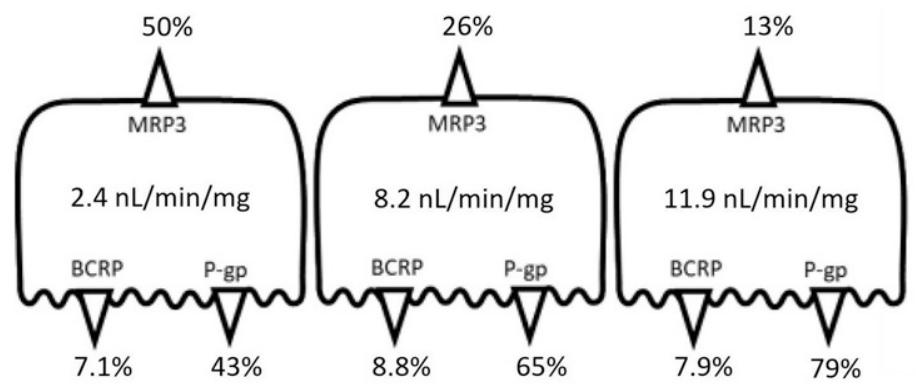

Fluvastatin

Pitavastatin

Rosuvastatin
Jejunum

Ileum
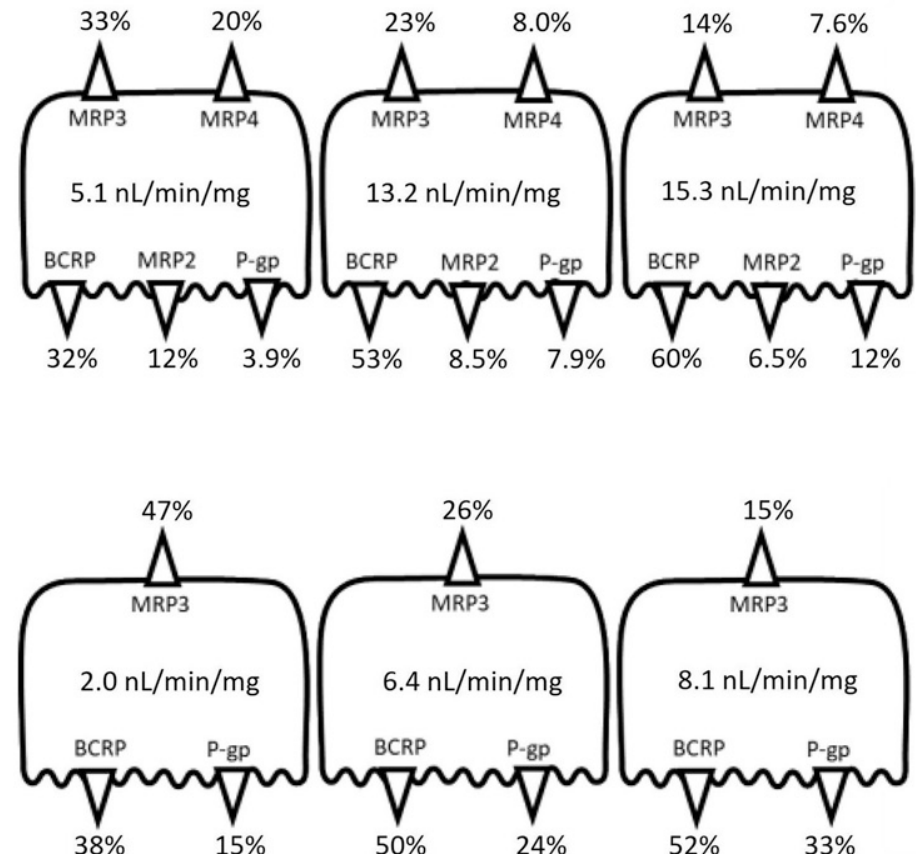

Liver
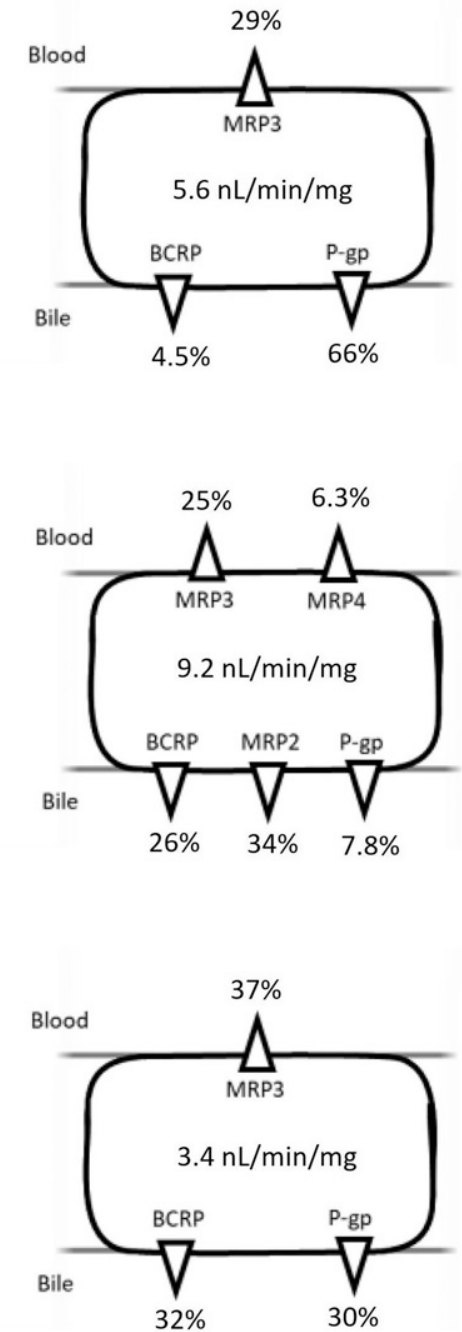

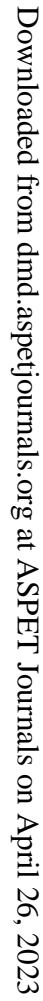

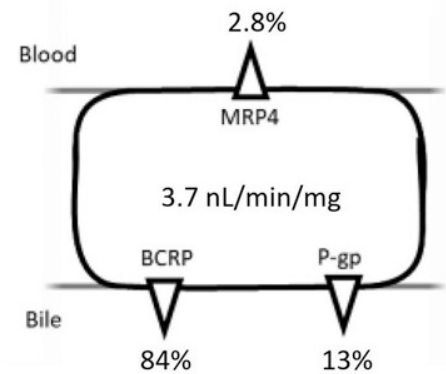

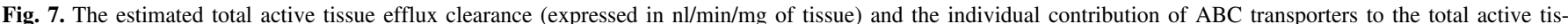

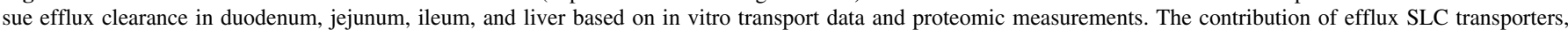
drug metabolism, and passive permeability on total clearance is not shown here.

in P-gp vesicles. The estimated tissue-specific efflux clearances indicate that P-gp, and BCRP to a smaller extent, could regulate atorvastatin plasma and tissue levels in humans. In line with this, previous studies have demonstrated increased systemic exposure to atorvastatin in association with genetic variants, which reduce the function or expression of the P-gp and BCRP (Keskitalo et al., 2008; Keskitalo et al., 2009c; León-Cachón et al., 2016). Moreover, our findings suggest that MRP3 may facilitate the intestinal absorption of atorvastatin especially in the jejunum and the hepatocyte hopping of atorvastatin and its metabolites. Moreover, MRP3 could be an important basolateral pathway for 2- 
hydroxyatorvastatin, which is extensively formed in the liver (Lau et al., 2006).

Fluvastatin is a relatively lipophilic statin but has a low bioavailability of $29 \%$ partly due to extensive first-pass hepatic metabolism (Tse et al., 1992). In the kinetic experiments, the highest transport rate and affinity were observed in BCRP vesicles, whereas the lowest affinity was seen in P-gp vesicles. We also demonstrated for the first time that fluvastatin is transported in MRP2, MRP3, MRP4, and MRP8 vesicles. Our scaled efflux clearance values suggest that the BCRP plays a major role in the apical efflux of fluvastatin in the intestine, whereas both MRP2 and BCRP may be important for the biliary clearance. In humans, a reduced-function genetic variant of BCRP was associated with increased exposure to fluvastatin (Keskitalo et al., 2009b). Furthermore, rat data support a role for MRP2 in fluvastatin biliary excretion (Lindahl et al., 2004), although observations in preclinical species must be treated with caution. Based on the scaled tissue efflux clearances, MRP3 and MRP4 could promote the intestinal absorption of fluvastatin. Together, MRP3 and MRP4 constitute $22 \%-53 \%$ of the total intestinal efflux clearance, depending on the site along the small intestine. In the hepatocytes, MRP3 and MRP4 may account for over $31 \%$ of the total efflux clearance and could thus enable fluvastatin hepatocyte hopping. Interestingly, fluvastatin was the sole substrate for MRP8 in our study. However, the impact of MRP8 remains elusive, since its membrane localization, abundance, and function are poorly characterized.

Pitavastatin is a lipophilic and metabolically stable statin that undergoes enterohepatic circulation, which explains its relatively high bioavailability of $51 \%$ and long half-life of 13 hours (Livalo LabelingPackage Insert., 2009; Saito, 2011). In the present study, BCRP and MRP3 transported pitavastatin with a similar affinity and scaled clearance, which was followed by P-gp with 2-fold lower affinity and clearance. Thus, we estimate that BCRP may comprise the majority of intestinal apical efflux, whereas BCRP and P-gp are equally important in its biliary clearance in hepatocytes. These estimations agree with the mechanistic modeling of pitavastatin disposition in sandwich-cultured human hepatocytes (SCHHs) (Vildhede et al., 2016). However, despite being a good BCRP substrate in vitro (Fujino et al., 2005; Hirano et al., 2005b), the pharmacokinetics of pitavastatin have not been affected by genetic variants of BCRP (Ieiri et al., 2007; Oh et al., 2013; Zhou et al., 2013). By contrast, a genetic variant of P-gp has been associated with increased $C_{\max }$ and AUC of pitavastatin (Zhou et al., 2013). The scaled MRP3-mediated efflux clearance exceeded that of BCRP by 2-fold in the liver and was equal in jejunum. This agrees with data from the earlier SCHH study (Vildhede et al., 2016). Therefore, MRP3 could facilitate pitavastatin absorption. In a previous study, the accumulation of pitavastatin into the liver was reduced in Mrp2-deficient rats (Hirano et al., 2005b). Interestingly, the expression of Mrp3 is induced in these rats, supporting the importance of Mrp3 in the intrahepatic distribution of pitavastatin (Ogawa et al., 2000; Kuroda et al., 2004).

Pravastatin is a hydrophilic statin with a low bioavailability (18\%) limited by acid-catalyzed biotransformation (Singhvi et al., 1990) and hepatic uptake (Niemi et al., 2006b). This is the first study to demonstrate that pravastatin is a substrate of MRP3. In fact, MRP3 was the only transporter, which transported pravastatin in our study. Our data may explain how pravastatin is transferred from enterocytes to the mesenteric vein, and effluxed from hepatocytes back to the circulation. Surprisingly, we observed no pravastatin transport in either human embryonic kidney-MRP2 or Sf9-MRP2 vesicles (Supplemental Fig. S9), even though previous studies have suggested that MRP2 is a key canalicular transporter for pravastatin (Niemi et al., 2006a; Nakagomi-Hagihara et al., 2007; Elsby et al., 2011). In Mrp2-deficient rats, pravastatin $\mathrm{C}_{\max }$ and AUC were increased compared with wild-type rats (Kivistö et al., 2005), which might be explained by the increased hepatic Mrp3 expression. Instead of MRP2, bile salt export pump could mediate the biliary excretion of pravastatin (Hirano et al., 2005a).

Rosuvastatin is another hydrophilic statin with a low bioavailability (20\%), minimal metabolism, and a long terminal half-life of 20 hours (Neuvonen et al., 2006). Of the statins tested in our study, BCRP transported rosuvastatin with the highest affinity and clearance. In contrast, minimal transport of rosuvastatin was observed in P-gp and MRP4 vesicles. Our findings suggesting an important role for BCRP in the hepatic and intestinal efflux of rosuvastatin are consistent with clinical data showing over 2-fold increase in the $\mathrm{C}_{\max }$ and $\mathrm{AUC}$ of rosuvastatin in healthy volunteers homozygous for a genetic defect in BCRP (Keskitalo et al., 2009c). The scaled clearance values imply that P-gp plays a limited role in rosuvastatin efflux, a finding supported by clinical pharmacogenetic data (Keskitalo et al., 2009a; Bai et al., 2019). Because of its hydrophilic nature, rosuvastatin may require basolateral efflux transporters in absorption and distribution. MRP4 could facilitate rosuvastatin absorption in the duodenum, where it was estimated to contribute $10 \%$ of rosuvastatin efflux clearance. Rat liver perfusion and $\mathrm{SCHH}$ studies have demonstrated significant basolateral efflux of rosuvastatin in the liver (Pfeifer et al., 2013a,b), but in our estimations, the role of hepatic MRP4 was minimal because of low in vitro activity and hepatic expression (Burt et al., 2016).

In conclusion, we compared the transport of atorvastatin, fluvastatin, pitavastatin, pravastatin, rosuvastatin, and simvastatin acid in BCRP, MRP2, MRP3, MRP4, MRP8, and P-gp vesicles. Using the vesicular transport assay and LC-MS/MS-based protein abundance measurements, we demonstrated the different efflux transport profiles and estimated the efflux clearances of statins in small intestine and liver. Because of assay variability in kinetic studies for certain statins and transporters, the estimated relative contributions of the different transporters to tissue efflux clearance should be interpreted with caution. Moreover, polarized-cell-monolayer-based or other alternative methods may be useful to confirm our findings. Overall, the present data can be applied to physiologically based pharmacokinetic or systems pharmacology modeling of statins.

\section{Acknowledgments}

The authors would like to thank Dr. Erkka Järvinen, Dr. Moshe Finel, and Dr. Heidi Kidron for providing the Sf9 vesicles.

\section{Authorship Contributions}

Participated in research design: Deng, Tuomi, Hirvensalo, Niemi. Conducted experiments: Deng, Tuomi, Neuvonen, Kulju, Wenzel, Oswald. Contributed new reagents or analytic tools: Neuvonen.

Performed data analysis: Deng, Tuomi, Oswald, Niemi.

Wrote or contributed to the writing of the manuscript: Deng, Tuomi, Neuvonen, Hirvensalo, Kulju, Wenzel, Oswald, Filppula, Niemi.

\section{References}

Bai X, Zhang B, Wang P, Wang GL, Li JL, Wen DS, Long XZ, Sun HS, Liu YB, Huang M, et al. (2019) Effects of SLCO1B1 and GATM gene variants on rosuvastatin-induced myopathy are unrelated to high plasma exposure of rosuvastatin and its metabolites. Acta Pharmacol Sin 40:492-499.

Bi YA, Costales C, Mathialagan S, West M, Eatemadpour S, Lazzaro S, Tylaska L, Scialis R, Zhang H, Umland J, et al. (2019) Quantitative contribution of six major transporters to the hepatic uptake of drugs: "SLC-phenotyping" using primary human hepatocytes. J Pharmacol Exp Ther 370:72-83.

Burt HJ, Riedmaier AE, Harwood MD, Crewe HK, Gill KL, and Neuhoff S (2016) Abundance of hepatic transporters in Caucasians: a meta-analysis. Drug Metab Dispos 44:1550-1561.

Deng F, Ghemtio L, Grazhdankin E, Wipf P, Xhaard H, and Kidron H (2020) Binding site interactions of modulators of breast cancer resistance protein, multidrug resistance-associated protein 2, and P-glycoprotein activity. Mol Pharm 17:2398-2410.

Drozdzik M, Busch D, Lapczuk J, Müller J, Ostrowski M, Kurzawski M, and Oswald S (2019) Protein abundance of clinically relevant drug transporters in the human liver and intestine: a comparative analysis in paired tissue specimens. Clin Pharmacol Ther 105:1204-1212. 
Elsby R, Hilgendorf C, and Fenner K (2012) Understanding the critical disposition pathways of statins to assess drug-drug interaction risk during drug development: it's not just about OATP1B1. Clin Pharmacol Ther 92:584-598.

Elsby R, Smith V, Fox L, Stresser D, Butters C, Sharma P, and Surry DD (2011) Validation of membrane vesicle-based breast cancer resistance protein and multidrug resistance protein 2 assays to assess drug transport and the potential for drug-drug interaction to support regulatory submissions. Xenobiotica 41:764-783.

Fujino H, Saito T, Ogawa S, and Kojima J (2005) Transporter-mediated influx and efflux mechanisms of pitavastatin, a new inhibitor of HMG-CoA reductase. $J$ Pharm Pharmacol 57:1305-1311.

Giacomini KM, Huang SM, Tweedie DJ, Benet LZ, Brouwer KL, Chu X, Dahlin A, Evers R, Fischer V, Hillgren KM, et al.; International Transporter Consortium (2010) Membrane transporters in drug development. Nat Rev Drug Discov 9:215-236.

Gröer C, Brück S, Lai Y, Paulick A, Busemann A, Heidecke CD, Siegmund W, and Oswald S (2013) LC-MS/MS-based quantification of clinically relevant intestinal uptake and efflux transporter proteins. J Pharm Biomed Anal 85:253-261.

Harper CR and Jacobson TA (2007) The broad spectrum of statin myopathy: from myalgia to rhabdomyolysis. Curr Opin Lipidol 18:401-408.

Hillgren KM, Keppler D, Zur AA, Giacomini KM, Stieger B, Cass CE, and Zhang L; Internationa Transporter Consortium (2013) Emerging transporters of clinical importance: an update from the International Transporter Consortium. Clin Pharmacol Ther 94:52-63.

Hirano M, Maeda K, Hayashi H, Kusuhara H, and Sugiyama Y (2005a) Bile salt export pump (BSEP/ABCB11) can transport a nonbile acid substrate, pravastatin. J Pharmacol Exp Ther 314:876-882.

Hirano M, Maeda K, Matsushima S, Nozaki Y, Kusuhara H, and Sugiyama Y (2005b) Involvement of BCRP (ABCG2) in the biliary excretion of pitavastatin. Mol Pharmacol 68:800-807.

Ieiri I, Suwannakul S, Maeda K, Uchimaru H, Hashimoto K, Kimura M, Fujino H, Hirano M, Kusuhara H, Irie S, et al. (2007) SLCO1B1 (OATP1B1, an uptake transporter) and ABCG2 (BCRP, an efflux transporter) variant alleles and pharmacokinetics of pitavastatin in healthy volunteers. Clin Pharmacol Ther 82:541-547.

Iusuf D, van de Steeg E, and Schinkel AH (2012) Hepatocyte hopping of OATP1B substrates contributes to efficient hepatic detoxification. Clin Pharmacol Ther 92:559-562.

Keskitalo JE, Kurkinen KJ, Neuvonen M, Backman JT, Neuvonen PJ, and Niemi M (2009a) No significant effect of $A B C B I$ haplotypes on the pharmacokinetics of fluvastatin, pravastatin, lovastatin, and rosuvastatin. Br J Clin Pharmacol 68:207-213.

Keskitalo JE, Kurkinen KJ, Neuvoneni PJ, and Niemi M (2008) ABCB1 haplotypes differentially affect the pharmacokinetics of the acid and lactone forms of simvastatin and atorvastatin. Clin Pharmacol Ther 84:457-461.

Keskitalo JE, Pasanen MK, Neuvonen PJ, and Niemi M (2009b) Different effects of the $A B C G 2$ c. $421 \mathrm{C}>\mathrm{A}$ SNP on the pharmacokinetics of fluvastatin, pravastatin and simvastatin. Pharmaco genomics 10:1617-1624

Keskitalo JE, Zolk O, Fromm MF, Kurkinen KJ, Neuvonen PJ, and Niemi M (2009c) ABCG2 polymorphism markedly affects the pharmacokinetics of atorvastatin and rosuvastatin. Clin Pharmacol Ther 86:197-203.

Kitamura Y, Kusuhara H, and Sugiyama Y (2010a) Basolateral efflux mediated by multidrug resistance-associated protein 3 (Mrp3/Abcc3) facilitates intestinal absorption of folates in mouse. Pharm Res 27:665-672.

Kitamura Y, Kusuhara H, and Sugiyama Y (2010b) Functional characterization of multidrug resistance-associated protein 3 (mrp3/abcc3) in the basolateral efflux of glucuronide conjugates in the mouse small intestine. J Pharmacol Exp Ther 332:659-666.

Kivistö KT, Grisk O, Hofmann U, Meissner K, Möritz K-U, Ritter C, Arnold KA, Lutjöohann D, von Bergmann K, Klöting I, et al. (2005) Disposition of oral and intravenous pravastatin in MRP2-deficient TR ${ }^{-}$rats. Drug Metab Dispos 33:1593-1596.

Kuroda M, Kobayashi Y, Tanaka Y, Itani T, Mifuji R, Araki J, Kaito M, and Adachi Y (2004) Increased hepatic and renal expressions of multidrug resistance-associated protein 3 in Eisai hyperbilirubinuria rats. J Gastroenterol Hepatol 19:146-153.

Lau YY, Okochi H, Huang Y, and Benet LZ (2006) Pharmacokinetics of atorvastatin and its hydroxy metabolites in rats and the effects of concomitant rifampicin single doses: relevance of first-pass effect from hepatic uptake transporters, and intestinal and hepatic metabolism. Drug Metab Dispos 34:1175-1181.

Lehtisalo M, Keskitalo JE, Tornio A, Lapatto-Reiniluoto O, Deng F, Jaatinen T, Viinamäki J, Neuvonen M, Backman JT, and Niemi M (2020) Febuxostat, but not allopurinol, markedly raises the plasma concentrations of the breast cancer resistance protein substrate rosuvastatin. Clin Transl Sci 13:1236-1243.

Lennernäs H (2003) Clinical pharmacokinetics of atorvastatin. Clin Pharmacokinet 42:1141-1160.

León-Cachón RBR, Ascacio-Martínez JA, Gamino-Peña ME, Cerda-Flores RM, Meester I, GallardoBlanco HL, Gómez-Silva M, Piñeyro-Garza E, and Barrera-Saldaña HA (2016) A pharmacogenetic pilot study reveals MTHFR, DRD3, and MDR1 polymorphisms as biomarker candidates for slow atorvastatin metabolizers. BMC Cancer 16:74.

Liao JK and Laufs U (2005) Pleiotropic effects of statins. Annu Rev Pharmacol Toxicol 45.89-118.
Lindahl A, Sjöberg A, Bredberg U, Toreson H, Ungell A-L, and Lennernäs H (2004) Regional intestinal absorption and biliary excretion of fluvastatin in the rat: possible involvement of mrp2. Mol Pharm 1:347-356.

Livalo Labeling-Package Insert. (2009). Kowa Pharm Am Inc

Maron DJ, Fazio S, and Linton MF (2000) Current perspectives on statins. Circulation 101:207-213.

Meszaros P, Klappe K, Hummel I, Hoekstra D, and Kok JW (2011) Function of MRP1/ABCC1 is not dependent on cholesterol or cholesterol-stabilized lipid rafts. Biochem J 437:483-491.

Nakagomi-Hagihara R, Nakai D, Tokui T, Abe T, and Ikeda T (2007) Gemfibrozil and its glucuronide inhibit the hepatic uptake of pravastatin mediated by OATP1B1. Xenobiotica 37:474-486.

Neuvonen PJ, Niemi M, and Backman JT (2006) Drug interactions with lipid-lowering drugs: mechanisms and clinical relevance. Clin Pharmacol Ther 80:565-581.

Niemi M, Arnold KA, Backman JT, Pasanen MK, Gödtel-Armbrust U, Wojnowski L, Zanger UM, Neuvonen PJ, Eichelbaum M, Kivistö KT, et al. (2006a) Association of genetic polymorphism in $\mathrm{ABCC} 2$ with hepatic multidrug resistance-associated protein 2 expression and pravastatin pharmacokinetics. Pharmacogenet Genomics 16:801-808.

Niemi M, Pasanen MK, and Neuvonen PJ (2006b) SLCO1B1 polymorphism and sex affect the pharmacokinetics of pravastatin but not fluvastatin. Clin Pharmacol Ther 80:356-366.

Niemi M, Pasanen MK, and Neuvonen PJ (2011) Organic anion transporting polypeptide 1B1: a genetically polymorphic transporter of major importance for hepatic drug uptake. Pharmacol $\operatorname{Rev~63:157-181.~}$

Ogawa K, Suzuki H, Hirohashi T, Ishikawa T, Meier PJ, Hirose K, Akizawa T, Yoshioka M, and Sugiyama Y (2000) Characterization of inducible nature of MRP3 in rat liver. Am J Physiol Gastrointest Liver Physiol 278:G438-G446.

Oh ES, Kim CO, Cho SK, Park MS, and Chung J-Y (2013) Impact of ABCC2, ABCG2 and SLCO1B1 polymorphisms on the pharmacokinetics of pitavastatin in humans. Drug Metab Pharmacokinet 28: 196-202

Oswald S, Müller J, Neugebauer U, Schröter R, Herrmann E, Pavenstädt H, and Ciarimboli G (2019) Protein abundance of clinically relevant drug transporters in the human kidneys. Int J Mol Sci 20:5303.

Pfeifer ND, Bridges AS, Ferslew BC, Hardwick RN, and Brouwer KLR (2013a) Hepatic basolateral efflux contributes significantly to rosuvastatin disposition II: characterization of hepatic elimination by basolateral, biliary, and metabolic clearance pathways in rat isolated perfused liver. J Pharmacol Exp Ther 347:737-745.

Pfeifer ND, Yang K, and Brouwer KLR (2013b) Hepatic basolateral efflux contributes significantly to rosuvastatin disposition I: characterization of basolateral versus biliary clearance using a novel protocol in sandwich-cultured hepatocytes. J Pharmacol Exp Ther 347:727-736.

Proctor WR, Ming X, Bourdet D, Han TK, Everett RS, and Thakker DR (2016) Why Does the intestine lack basolateral efflux transporters for cationic compounds? A provocative hypothesis. J Pharm Sci 105:484-496.

Roth GA, Johnson C, Abajobir A, Abd-Allah F, Abera SF, Abyu G, Ahmed M, Aksut B, Alam T, Alam K, et al. (2017) Global, regional, and national burden of cardiovascular diseases for 10 causes, 1990 to 2015. J Am Coll Cardiol 70:1-25.

Saito Y (2011) Pitavastatin: an overview. Atheroscler Suppl 12:271-276.

Singhvi SM, Pan HY, Morrison RA, and Willard DA (1990) Disposition of pravastatin sodium, a tissue-selective HMG-CoA reductase inhibitor, in healthy subjects. $\mathrm{Br} \mathrm{J}$ Clin Pharmacol 29:239-243.

Slater EE and MacDonald JS (1988) Mechanism of action and biological profile of HMG CoA reductase inhibitors. A new therapeutic alternative. Drugs 36 (Suppl 3):72-82.

Tse FLS, Jaffe JM, and Troendle A (1992) Pharmacokinetics of fluvastatin after single and multiple doses in normal volunteers. J Clin Pharmacol 32:630-638.

van de Wetering K, Burkon A, Feddema W, Bot A, de Jonge H, Somoza V, and Borst P (2009) Intestinal breast cancer resistance protein (BCRP)/Bcrp1 and multidrug resistance protein 3 (MRP3)/Mrp3 are involved in the pharmacokinetics of resveratrol. Mol Pharmacol 75:876-885.

Vildhede A, Mateus A, Khan EK, Lai Y, Karlgren M, Artursson P, and Kjellsson MC (2016) Mechanistic modeling of pitavastatin disposition in sandwich-cultured human hepatocytes: a proteomics-informed bottom-up approach. Drug Metab Dispos 44:505-516.

Yebyo HG, Aschmann HE, Kaufmann M, and Puhan MA (2019) Comparative effectiveness and safety of statins as a class and of specific statins for primary prevention of cardiovascular disease: A systematic review, meta-analysis, and network meta-analysis of randomized trials with 94,283 participants. Am Heart J 210:18-28.

Zhou Q, Chen QX, Ruan Z-R, Yuan H, Xu H-M, and Zeng S (2013) CYP2C9*3(1075A > C), $\mathrm{ABCB} 1$ and SLCO1B1 genetic polymorphisms and gender are determinants of inter-subject variability in pitavastatin pharmacokinetics. Pharmazie 68:187-194.

Address correspondence to: Mikko Niemi, Department of Clinical Pharmacology, PO Box 20 (Tukholmankatu 8 C), 00014 University of Helsinki, Finland. E-mail: mikko.niemi@helsinki.fi 